\title{
Noncontact simultaneous dual wavelength photoplethysmography: A further step toward noncontact pulse oximetry
}

\author{
Kenneth Humphreys and Tomas Ward \\ Department of Electronic Engineering, National University of Ireland, Maynooth, Maynooth Co. \\ Kildare, Ireland \\ Charles Markham \\ Department of Computer Science, National University of Ireland, Maynooth, Maynooth Co. Kildare, Ireland
}

(Received 22 December 2006; accepted 19 March 2007; published online 23 April 2007)

\begin{abstract}
We present a camera-based device capable of capturing two photoplethysmographic (PPG) signals at two different wavelengths simultaneously, in a remote noncontact manner. The system comprises a complementary metal-oxide semiconductor camera and dual wavelength array of light emitting diodes $(760$ and $880 \mathrm{~nm}$ ). By alternately illuminating a region of tissue with each wavelength of light, and detecting the backscattered photons with the camera at a rate of 16 frames/wavelength s, two multiplexed PPG wave forms are simultaneously captured. This process is the basis of pulse oximetry, and we describe how, with the inclusion of a calibration procedure, this system could be used as a noncontact pulse oximeter to measure arterial oxygen saturation $\left(S_{p} \mathrm{O}_{2}\right)$ remotely. Results from an experiment on ten subjects, exhibiting normal $S_{p} \mathrm{O}_{2}$ readings, that demonstrate the instrument's ability to capture signals from a range of subjects under realistic lighting and environmental conditions are presented. We compare the signals captured by the noncontact system to a conventional PPG signal captured concurrently from a finger, and show by means of a J. Bland and D. Altman [Lancet 327, 307 (1986); Statistician 32, 307 (1983)] test, the noncontact device to be comparable to a contact device as a monitor of heart rate. We highlight some considerations that should be made when using camera-based "integrative" sampling methods and demonstrate through simulation, the suitability of the captured PPG signals for application of existing pulse oximetry calibration procedures. (C) 2007 American Institute of Physics. [DOI: 10.1063/1.2724789]
\end{abstract}

\section{INTRODUCTION}

Pulse oximetry ${ }^{1-4}$ (the noninvasive measuring of arterial oxygen saturation) has, since its invention by Aoyagi in $1973,{ }^{5}$ become a crucial clinical tool for monitoring the health of critically ill, anaesthetized, and recovering patients. ${ }^{1}$ It is primarily employed as the earliest warning of hypoxic hypoxia and has become a standard of care in operating and recovery rooms in many countries. A conventional pulse oximeter sensor consists of two light emitting diodes with different peak emission wavelengths, typically $660 \mathrm{~nm}$ (red) and $940 \mathrm{~nm}$ (infrared), ${ }^{6-8}$ and a photodiode detector, housed in a probe that is attached around a patient's finger. The light emitting diodes (LED) are alternately energized and the transmitted light passes through the tissue and is detected by the photodiode in a multiplexed fashion. Since red blood cells, or more specifically the oxygen carrying molecule hemoglobin present on red blood cells, is the principal absorber in tissue at these wavelengths, the received light intensity at the photodiode is largely proportional to the absorption by blood. The wavelengths of the LEDs are chosen to have maximum absorption by deoxygenated hemoglobin $(\mathrm{Hb})$ at the red wavelength, and oxygenated hemoglobin $\left(\mathrm{HbO}_{2}\right)$ at the infrared wavelength, thus the ratio of absorption at the two wavelengths is proportional to the concentrations of $\mathrm{Hb}$ and $\mathrm{HbO}_{2}$, and hence oxygen saturation. Where light from the LEDs has passed through an artery, the re- ceived light intensity contains a photoplethysmogram (PPG) at each wavelength, which is the modulation in absorptivity caused by the peripheral arterial pressure wave form. The PPG contains a dc offset due to absorption by venous blood, other tissues, and scattering losses. Pulse oximeters extract the PPG signal at each wavelength and by comparing the logarithms of the ratios, of peaks and valleys, and of corresponding pulses from each PPG, one produces a variable called the ratio of ratios $\left(R_{O S}\right)$ according to ${ }^{2,3}$

$$
R_{O S}=\frac{\ln \left(R_{L} / R_{H}\right)}{\ln \left(\mathrm{IR}_{L} / \mathrm{IR}_{H}\right)} .
$$

The subscripts $L$ and $H$ denote the valleys and peaks, respectively, of the red $(R)$ and infrared (IR) PPG signals. Since $R_{O S}$ is obtained from the PPG signals only, it is proportional to the variable of clinical interest, the arterial oxygen saturation $\left(S_{p} \mathrm{O}_{2}\right)$, which is defined as ${ }^{1}$

$$
S_{p} \mathrm{O}_{2}=\frac{\mathrm{HbO}_{2}}{\mathrm{HbO}_{2}+\mathrm{Hb}} \times 100 \% \text {. }
$$

An empirically derived calibration table, specific to the precise two wavelengths used, maps the $R_{O S}$ variable to an $S_{p} \mathrm{O}_{2}$ arterial oxygen saturation percentage. ${ }^{3}$

In addition, clinical pulse oximeters monitor and display the patient's heart rate, using the PPG wave form, and display a plethysmograph trace. The plethysmogram is used to give a quick indication of the cardiac rhythm and any 
changes in its regularity. ${ }^{1}$ When the displayed trace is not normalized, the plethysmogram will indicate sudden changes in cardiac output or hypovolemia, which is an important function of a clinical device that may otherwise continue displaying valid $S_{p} \mathrm{O}_{2}$ values, despite the diminishing PPG amplitude. ${ }^{1}$ Where the plethysmogram is completely unprocessed the arterial wave form may be seen superimposed on the slower respiratory wave form, though the amplitude of this wave form varies with intrathoracic and central venous pressure. $^{9}$

Motivated by trends towards remote sensing in general, the desire to reduce the physical restrictions and cabling associated with patient monitoring, removal of potentially injurious wires from magnetic resonance imaging (MRI) machines, ${ }^{10-12}$ and the possible new insights that might come from haemodynamic imaging and mapping, researchers have been working towards noncontact, remote camera-based pulse oximetry. The feasibility of a remote camera-based approach has been well demonstrated. We have previously reported the capture of PPG wave forms from extremities at one wavelength in a transmission modality at $810 \mathrm{~nm}$ using a complementary metal-oxide semiconductor (CMOS) camera. ${ }^{13}$ Wieringa et al. reported the reflection mode capture of "heartcycle-related" pulsatile variations also using a CMOS camera. ${ }^{14}$ They employed illumination wavelengths of 660, 810, and $940 \mathrm{~nm}$; however, simultaneous capture was not demonstrated. We have also previously reported the first simultaneous capture of PPG signals at two wavelengths (760 and $880 \mathrm{~nm}$ ) in both transmission and reflection modalities. ${ }^{15,16}$ However, the reflection mode PPG signals were weak and unsuited to the application of Eq. (1) for the derivation of conventional pulse oximeter calibration tables. Instead we applied the modified Beer-Lambert law as a set of simultaneous equations to estimate the concentrations of $\mathrm{Hb}$ and $\mathrm{HbO}_{2}$. This method is common in studies of tissue oxygenation but is impractical for a clinical device, as it requires the estimation of the optical path length and differential path length factor for both wavelengths, for each subject and for each observation location. ${ }^{17-19}$ In addition the system was limited to small extremities (typically fingers) where arteries are close to the surface.

In this article we present a system capable of capturing two PPG signals at two different wavelengths simultaneously and in a remote noncontact manner. We present results from an experiment on ten subjects and show the system to be capable of obtaining good quality PPG signals from deep tissue, and evaluate the comparability of the device with a conventional contact instrument.

\section{METHODS}

\section{A. Instrumentation}

The main components of the imaging system are depicted in Fig. 1. The camera (a monochromatic Pixelink PLA741) uses a 2/3 in. CMOS sensor with 1280 $\times 1024$ square pixels of length $6.7 \mu \mathrm{m}$. Pixels are encoded using 8 bits. The camera is connected to a personal compupter (PC) via an IEEE 1394 connection. The camera is manually focused on the tissue under investigation by a
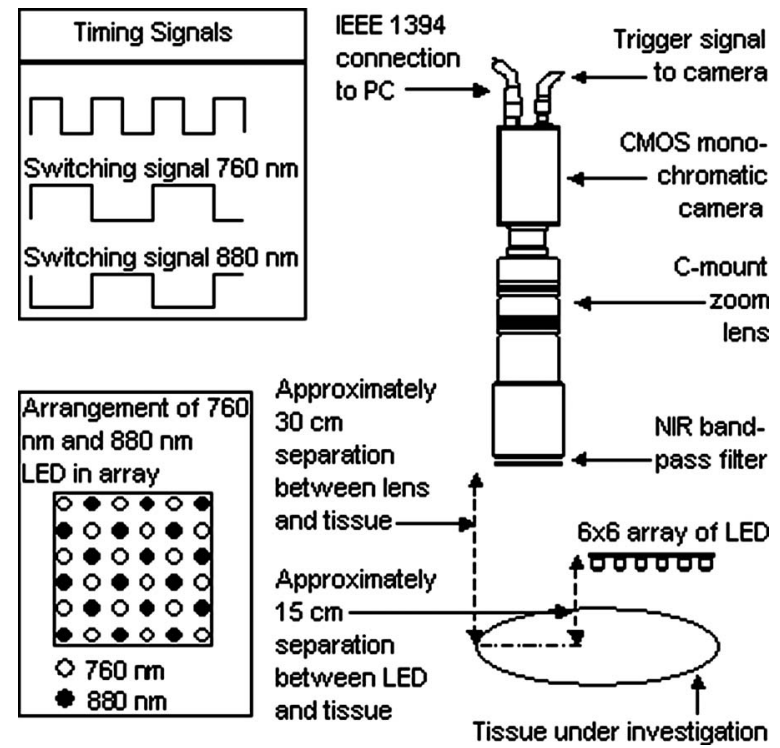

FIG. 1. Illustration of noncontact dual wavelength PPG system, depicting illumination and imaging geometry, relative timing of events (upper inset), and arrangement of LED array (lower inset).

C-mount zoom lens (focal length of $18-108 \mathrm{~mm}$, f2.5-closed). A NIR bandpass glass filter is mounted directly in front of the lens. Upon receiving a trigger, the camera exposes all pixels within a user specified region of interest for the desired exposure time and then applies a global shutter, such that all pixels are exposed for the same duration. Captured files are saved to the PC as a video file in uncompressed AVI format. The camera is mounted on a tripod with the lens face approximately $30 \mathrm{~cm}$ from the surface of the tissue under investigation.

The light source is positioned perpendicular to the tissue surface at a distance of approximately $15 \mathrm{~cm}$. The source is composed of 36 LED-18 with a peak wavelength of $760 \mathrm{~nm}$ and 18 with a peak wavelength of $880 \mathrm{~nm}^{20}$ The arrangement of the 760 and $880 \mathrm{~nm}$ LEDs is depicted in the lower inset of Fig. 1. The diodes used are metal cased with built-in glass lenses and have a half intensity beam angle of $30^{\circ}$. The $880 \mathrm{~nm}$ diodes (Opto Diode Corp. OD880-L) emit $17 \mathrm{~mW}$ at a forward current of $100 \mathrm{~mA}$. The $760 \mathrm{~nm}$ diodes (Opto Diode Corp. custom wavelength L-package) emit $10 \mathrm{~mW}$ at the same forward current. At 880 and $760 \mathrm{~nm}$ the camera has spectral sensitivities of 0.055 and $0.091 \mathrm{~A} / \mathrm{W}$, respectively. A control circuit alternately energizes each of the wavelengths on the array, such that the array alternately produces equal duration pulses of diffuse light centered at 760 and $880 \mathrm{~nm}$. As either wavelength on the array is being energized, the camera receives a trigger signal and begins integration of a frame. The relative timing of the signal is illustrated in the upper inset of Fig. 1. In order to identify the peaks and troughs of the of the multiplexed PPG wave forms as accurately as possible, it is desirable to use the highest possible frame rate. The maximum achievable frame rate is determined by the exposure time of a frame and the associated readout time, which is proportional to the frame size. The minimum required exposure time is determined experimentally as the shortest exposure time that results in sufficiently well defined PPG signals. To eliminate any error due 
(A)

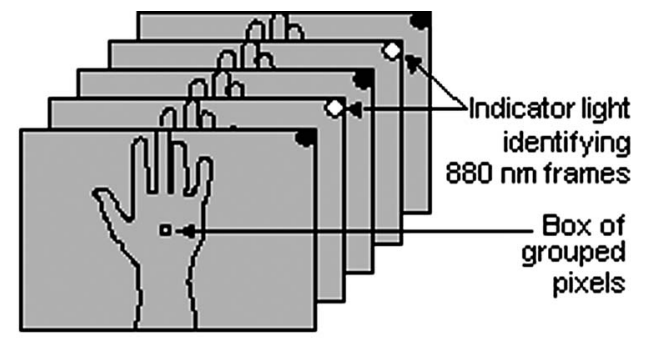

(日)
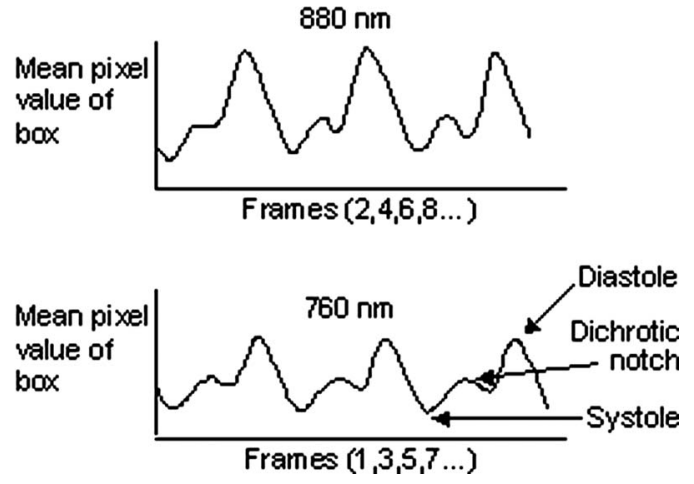

FIG. 2. Signal acquisition and image processing.

to dropped frames an indicator fiber coupled to an $880 \mathrm{~nm}$ LED provides a synchronization signal as part of the camera image.

The illumination scheme used is depicted in Fig. 1. The LED array is positioned to shine perpendicularly onto an area of tissue to one side of the camera's field of view. The camera images a comparatively poorly illuminated area of tissue adjacent to the brightly illuminated area. NIR light from a point source will scatter throughout the surrounding tissue; however, it has been shown theoretically and experimentally ${ }^{21}$ that backscattered light detected on the tissue surface is most likely to have traveled along a curved "banana-shaped" path through the tissue, the depth of the curve being proportional to the separation between the source and detector. In addition to light incident from the array and light reflected from the surface, the camera captures some light that has passed through the tissue. In some regions of the camera's field of view, backscattered light will have passed through an artery and have been modulated by the pulsing blood flow.

\section{B. Signal acquisition and processing}

Recorded videos are processed offline using MATLAB ${ }^{\circ}$. By searching for the presence (or absence) of the indicator light, videos are split into two sequences of frames; one illuminated at $760 \mathrm{~nm}$ and the other at $880 \mathrm{~nm}$, each sequence being half the original sampling rate. The process is illustrated in Fig. 2. Each frame is divided into boxes or groups of adjacent pixels (of dimensions $40 \times 40$ pixels). The average value of the pixels in each box is tracked from frame to frame as depicted in Fig. 2(a). This process is performed for both sequences of frames. Where backscattered light emerging from a box has passed through an artery, a PPG signal can be detected in the time-varying mean value of that box as

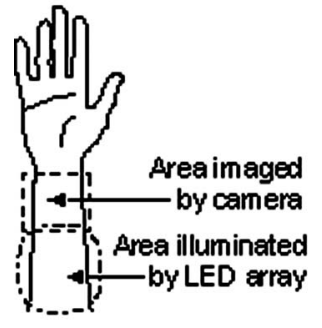

(A)

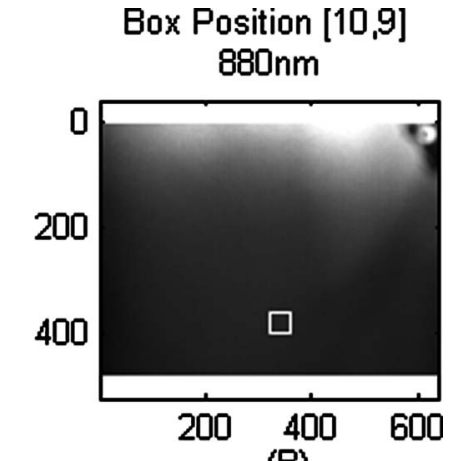

(B)

FIG. 3. Illustration of illumination and imaging positions (A) and sample frame from the $880 \mathrm{~nm}$ sequence (B). The white box encloses an area of $40 \times 40$ pixels - the time varying mean value of which is plotted in Fig. 4 .

in Fig. 2(b). This process yields two multiplexed PPG signals, captured at two wavelengths simultaneously-the prerequisite for pulse oximetry.

\section{Experimental protocol}

Experiments were conducted using ten healthy subjects (10 Caucasian, 9 male, and 1 female) aged between 21 and 45 years (mean 31.70 years and standard deviation 6.37 years). Subjects were seated in an upright position with their right arms resting, volar side upwards, on a cushioned bench. A conventional reflection mode contact PPG sensor (BioPac UIM100C) was connected to the subjects' right index fingers, and a conventional transmission mode contact pulse oximeter sensor (Nellcor probe/Welch Allyn pulse oximeter) to their left index fingers. The camera was positioned and focused such that an area of the volar side of the forearm close to the wrist was in view and occupying most of the image. The LED array was positioned so as to illuminate an area on the proximal side of the imaged area as illustrated in Fig. 3(a). Overhead fluorescent lighting was present during the experiments (main supply $50 \mathrm{~Hz}$ ). Once stable pulse rate and $S_{p} \mathrm{O}_{2}$ readings were indicated by the pulse oximeter, a trigger signal was sent simultaneously to the camera system and the PPG data acquisition system, initiating a $20 \mathrm{~s}$ capture of data by both devices. The camera was configured to capture 640 frames, at a resolution of $640 \times 480$ pixels, with a $20 \mathrm{~ms}$ exposure time. The camera was triggered externally at a rate of 32 frames/s and the illumination array correspondingly switched LED wavelengths at $16 \mathrm{~Hz}$. The LED array was activated prior to each experiment to allow the diodes reach their stable operating temperature and wavelengths. ${ }^{22}$ The subjects were asked to sit motionless and to breathe normally during the experiments.

\section{TESTING AND PERFORMANCE}

To successfully replace a conventional device in any application, a noncontact pulse oximeter should perform comparably the three main functions of a pulse oximeter, namely, measure $S_{p} \mathrm{O}_{2}$, measure heart rate, and display a plethysmogram. ${ }^{1}$ For clinical purposes the word "comparably" means that measurements by the two instruments should 

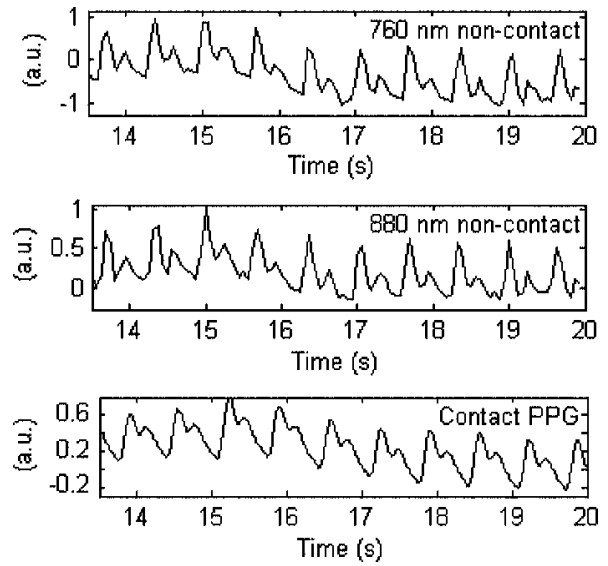

FIG. 4. Comparison of noncontact PPG signals and conventional PPG signal.

agree sufficiently closely that actions and decisions regarding the patient would not change depending on the instrument used. We present an analysis of the comparability of the contact and noncontact devices, with respect to the three primary functions of a clinical pulse oximeter, using graphical and numerical techniques as appropriate.

\section{A. Display a plethysmogram}

The results of a typical experiment are presented here. Figure 3(a) illustrates the relative position of the imaged and illuminated areas of tissue. Figure 3(b) displays a sample frame from the $880 \mathrm{~nm}$ frame sequence. Imposed on the sample frame is a white square (of dimensions 40 $\times 40$ pixels). [Also visible in the top right corner of Fig. 3(b) is the edge of the indicator fiber.] The mean values of the pixels contained in the white square are plotted against time in Fig. 4. (Note that the camera derived wave forms have been inverted so that their vertical axes correspond to light absorption rather than received light intensity; light absorption being directly proportional to the peripheral arterial pressure wave form; the received light intensity being inversely proportional.) The systolic peaks and diastolic troughs, along with the dichrotic notch (the inflection in the wave form associated with a momentary backflow of blood caused by the abrupt closure of the aortic valve), are clearly visible. The Fourier spectra of the three PPG signals in Fig. 4 are plotted in Fig. 5. The pulsatile component is clearly visible in all three spectra at $1.5 \mathrm{~Hz}$. The component due to respiration is clear in both noncontact signals at $0.35 \mathrm{~Hz}$, though is less pronounced in the contact PPG spectrum from this subject. The low-frequency vasomotion and Mayer wave oscillations are present in all three spectra as distinct peaks between 0 and $0.1 \mathrm{~Hz}$.

\section{B. Measure heart rate}

Here we assess the agreement between the contact and noncontact measurements of heart rate using the BlandAltman method. ${ }^{23,24}$ This is a popular method of assessing agreement between two clinical devices, where both devices exhibit a certain amount of measurement error. It is preferred to alternative methods, most notably the use of the correla-
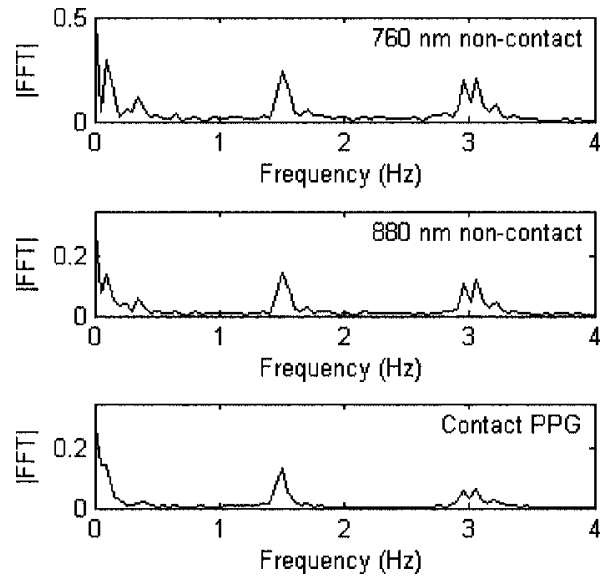

FIG. 5. Comparison of Fourier spectra of noncontact and conventional PPG signals.

tion coefficient, as it identifies bias between the devices. To obtain a heart rate measurement for each subject over the 20 s observation window, the Fourier spectra of the contact, 760 and $880 \mathrm{~nm}$ PPG signals were calculated and the peak frequency of the cardiac component noted. Figure 6 shows a scatter plot of the heart rate measurement from the contact device versus the measurement from the noncontact device. The noncontact $760 \mathrm{~nm}$ measurements are plotted as circles and the $880 \mathrm{~nm}$ measurements as crosses. Where the circles and crosses align the noncontact measurements agree at both wavelengths, for that subject. Also plotted in Fig. 6 is the line $x=y$, where data points lie along this line there is perfect agreement between the two devices, while deviation from the line indicates disagreement. (It is worth noting there will be perfect correlation between the devices if the points lie along any straight line.) It can be seen in Fig. 6 that for each subject the noncontact device indicates exactly the same measurement of heart rate at 760 and $880 \mathrm{~nm}$. For eight subjects, both the contact and noncontact devices are in perfect agreement; however, for two subjects the noncontact device recorded a slightly lower heart rate than the contact device. Since the two devices are in agreement for higher, intermediate, and lower heart rates, and since both devices agree for other heart rate measurements in this range, we can conclude

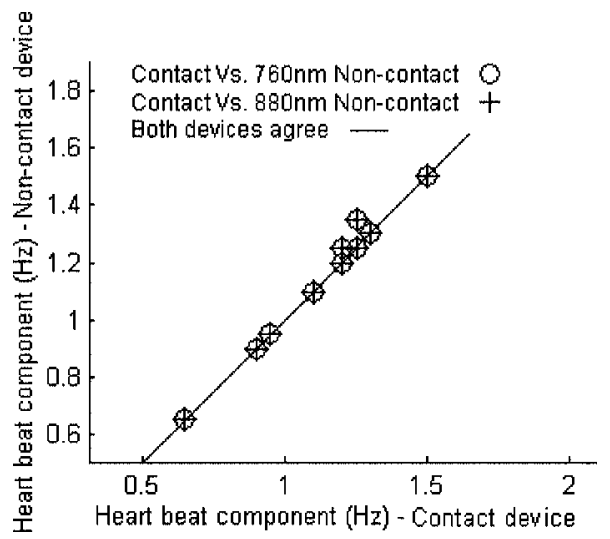

FIG. 6. Scatter plot showing noncontact device's measurement of heart rate at $760 \mathrm{~nm}$ (circles) and $880 \mathrm{~nm}$ (crosses), vs the contact device's measurement, for each subject. Also shown is the line $x=y$, where both devices are in perfect agreement about the measurement. 


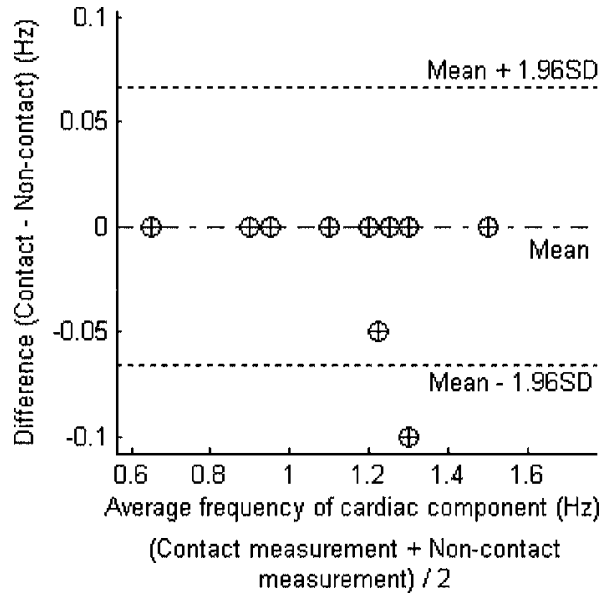

FIG. 7. Bland-Altman (mean vs difference) plot, showing the average of the heart rate measurements by the contact and noncontact devices, vs the difference between their measurements, for each subject. (The circles indicate measurement at $760 \mathrm{~nm}$, and the crosses at $880 \mathrm{~nm}$.) Also plotted are lines indicating the mean of the differences and \pm 1.96 standard deviations about the mean.

that the disagreement for these two subjects is not systemic to either device.

Figure 7 shows a Bland-Altman plot of the mean of the measurements by both devices for each subject versus the difference between the measurements by both devices for each subject. Again data pertaining to $760 \mathrm{~nm}$ are represented by circles and $880 \mathrm{~nm}$ by crosses. If the two devices disagreed more, this plot would allow us to identify any relationship between the measurement error and the true value of the variable of interest (of which, the mean of the two measurements is the best estimate we have). For these subjects the mean difference between the measurements by each device is $-0.0150 \mathrm{~Hz}$ and the standard deviation (SD) is $0.0337 \mathrm{~Hz}$. Assuming that the mean difference between the devices is normally distributed, the $95 \%$ confidence interval can be calculated at $\pm 1.96 \mathrm{SD}$ (though it would take many more samples of the measurement difference between the devices to establish if the difference is normally distributed, and a histogram would show that these samples are not). In this case the \pm 1.96 SD range means that the noncontact device may indicate a heart rate of 4.8687 beats $/ \mathrm{min}$ below or $3.0687 \mathrm{beats} / \mathrm{min}$. above the contact device, which is sufficiently close for the two devices to be used interchangeably.

\section{Measure $\mathrm{S}_{p} \mathrm{O}_{2}$ (ratio of ratios)}

Pulse oximeters calculate $S_{p} \mathrm{O}_{2}$ by way of an interim variable $R_{O S}$ and then employ empirically derived calibration curves to convert $R_{O S}$ to $S_{p} \mathrm{O}_{2}$. In addressing the suitability of noncontact PPG signals for use with existing methods of determining $R_{O S}$ (such as the peak and valley method or the derivative method) ${ }^{3}$ two points about camera-based systems should be noted that have not yet been reported in studies on the feasibility of such systems. The first is the effect of low frame rates. Pulse oximeters typically sample at $480 \mathrm{~Hz}$, sampling each wavelength and a dark sample in succession. ${ }^{7}$ Camera-based systems will never enjoy these frame rates; however, at very low rates (as low as 6.7 frames/s have been reported) the error in estimating the amplitude of the PPG
TABLE I. Simulated percentage error in measurement of $R_{O S}$ by integrative sampling (camera based) compared to sample-and-hold (contact method). Results pertain to a synthetic contact signal sampled at $160 \mathrm{~Hz}$ sample-andhold method and the camera derived version of that signal at 16 frames/s.

\begin{tabular}{ccccc}
\hline \hline \multirow{2}{*}{$\begin{array}{c}\text { Approx. } \\
\mathrm{S}_{p} \mathrm{O}_{2} \\
(\%)\end{array}$} & IR:R PPG & \multicolumn{3}{c}{ Pulse rate } \\
\cline { 3 - 5 } & scaling factor & 50 & 72 & 100 \\
\hline \multirow{2}{*}{100} & 2.32 & 1.3 & 1.69 & 2.58 \\
& 1.66 & 0.69 & 0.9 & 1.35 \\
85 & 1 & 0 & 0 & 0 \\
& 0.81 & -0.2 & -0.28 & -0.4 \\
60 & 0.47 & -0.51 & -0.73 & -1.09 \\
\hline \hline
\end{tabular}

peaks and valleys that can result from sampling the wave form before or after the actual inflection are significant. Second, this problem is compounded by the way a camera collects its data. The photodiode in a contact device produces a continuously varying output current that is converted to a voltage and sampled by a sample-and-hold circuit once every 2 ms. The camera exposes its CMOS or charge-coupled device (CCD) chip for typically tens of milliseconds and then has a short readout time before beginning the next exposure. However, the value readout at the end of the exposure is not an instantaneously sampled value but rather a charge proportional to the sum of all photons received during the exposure - that is, the camera derived signal is proportional to the mean value observed during exposure. This integration effect means the camera produces an underestimation when the wave form has a positive slope and an overestimation when the wave form has a negative slope. Thus the cameraderived wave form appears to lag the sample-and-hold wave form and has reduced peak-to-valley amplitude.

To quantify the effect of this phenomenon on the calculation of $R_{O S}$, we simulated the camera integration effect (at 16 frames/wavelength s) on a synthetic PPG wave form (constructed by summing three sinusoids at typical cardiac, respiratory, and vasomotion frequencies) and investigated the variation in $R_{O S}$ values between the sample-and-hold and integrated wave forms, for varying oxygen saturation levels and heart rates. Changes in oxygen saturation levels were emulated by altering the IR:red PPG amplitudes between approximately 2.3 and 0.5 , which for wavelengths of 660 and $940 \mathrm{~nm}$ would correspond to $S_{p} \mathrm{O}_{2}$ levels between approximately $100 \%$ and $60 \%{ }^{1,2} R_{O S}$ was calculated using Eq. (1). The results are summarized in Table I. The discrepancy between $R_{O S}$ calculated from the sample-and-hold wave form and from the integrated wave form varies with both heart rate and oxygen saturation. The difference is largest when the heart rate and oxygen saturation are at their highest. At lower heart rates the simulated PPG has smaller slopes and the integration effect is less pronounced. Similarly as the ratio of the infrared and red PPG amplitudes varies with oxygen saturation, the quotient of the integration error for each wavelength also varies-canceling itself out when the PPG wave forms have similar amplitudes. Within the range of heart rate and oxygen saturation values simulated, compared to the contact technique, the noncontact technique produced a maximum overestimation of $R_{O S}$ of $2.6 \%$ and maximum 
underestimation of $-1.1 \%$. The $S_{p} \mathrm{O}_{2}$ measurement error associated with a conventional pulse oximeter is approximately $\pm 3 \%$. ${ }^{3,25}$ If the $R_{O S}$ differences summarized in Table I were translated directly in the $S_{p} \mathrm{O}_{2}$ measurement differences, the noncontact device might not be comparable with a conventional device at high levels of oxygen saturation and particularly for high heart rates. At lower levels of oxygen saturation the differences between devices are less than the $\pm 3 \%$ measurement error associated with conventional pulse oximetry.

\section{DISCUSSION}

Though obstacles to developing a clinically useful device remain, the allure of a noncontact pulse oximeter and the ability to measure $S_{p} \mathrm{O}_{2}$ and heart rate and observe a PPG by noncontact means are strong. Such an instrument will not compete with conventional devices for routine bedside monitoring or for ambulatory monitoring, but would most likely find application in specialized areas such as sleep studies, intensive care, or in conjunction with other modalities such as MRI.

The instrument presented here performs comparably with a conventional contact device as a measure of heart rate and as a capturing system for the PPG wave form. We have drawn attention to the effect of an integrative sampling technique (inherent to all camera-based approaches) as compared to a conventional sample-and-hold method. While this is something to consider when converting $R_{O S}$ values to $S_{p} \mathrm{O}_{2}$ values, the simulation results indicate that interdevice differences may be clinically significant only at very high oxygen saturations - though the necessity to ascertain precise $S_{p} \mathrm{O}_{2}$ measurements in this region is less critical than at lower saturations, where a patient may be in danger. ${ }^{1}$ The ultimate solution to this is higher frame rates though it may also be possible to account for integrative effects during the calibration procedure or by applying time-domain system identifi- cation techniques to the captured PPG signals. We contend, however, that the recorded PPG signals are suitable for the application of existing pulse oximetry calibration procedures to convert PPG data to $S_{p} \mathrm{O}_{2}$ values, which would yield full pulse oximeter functionality through noncontact means.

${ }^{1}$ J. Moyle, Pulse Oximitery (BMJ P, London, 1994).

${ }^{2}$ J. Payne and J. Severinghaus, Pulse Oximetry (Springer, New York, 1986).

${ }^{3}$ J. Webster, Design of Pulse Oximeters (Institute of Physics, Bristol, 1997).

${ }^{4}$ J. Kelleher, J. Clin. Monit 5, 37 (1989).

${ }^{5}$ T. Aoyagi, J. Anesth. 17, 259 (2003).

${ }^{6} \mathrm{M}$. Wukitsch, M. Petterson, D. Tobler, and J. Pologe, J. Clin. Monit 1988, 290 (1988).

${ }^{7}$ M. Wukitsch, J. Clin. Monit Comput. 4, 161 (1987).

${ }^{8}$ J. Severinghaus, Adv. Exp. Med. Biol. 220, 3 (1987).

${ }^{9}$ J. Dorlas and J. Nijboer, Br. J. Anesth. 57, 524 (1985).

${ }^{10}$ T. Nakamura, K. Fukuda, K. Hayakawa, I. Aoki, K. Matasumoto, T. Senkine, H. Ueda, and Y. Shimizu, Front Med. Biol. Eng. 11, 117 (2001).

${ }^{11}$ F. Shellock, AJR, Am. J. Roentgenol. 153, 1105 (1989).

${ }^{12}$ T. Brown, B. Goldstein, and J. Little, Am. J. Phys. Med. Rehabil. 72, 166 (1993).

${ }^{13}$ K. Humphreys, C. Markham, and T. Ward, Proc. SPIE 5823, 88 (2005).

${ }^{14}$ F. Wieringa, F. Mastik, and A. Van Der Steen, Ann. Biomed. Eng. 33, 1034 (2005).

${ }^{15} \mathrm{~K}$. Humphreys, T. Ward, and C. Markham, Annual International Conference of the IEEE Engineering in Medicine and Biology Society, Vol. 4, p. 3494 (2005).

${ }^{16} \mathrm{~K}$. Humphreys, T. Ward, and C. Markham, Proceedings of the International Federation for Medical and Biological Engineering, Vol. 11 (2005).

${ }^{17}$ C. Elwell, A Practical Users Guide to Near Infrared Spectroscopy (UCL Reprographics, London, 1995).

${ }^{18}$ P. Rolfe, Annu. Rev. Biomed. Eng. 2, 715 (2000).

${ }^{19}$ A. Duncan, J. Meek, M. Clemence, C. Elwell, L. Tyszczuk, M. Cope, and D. Deply, Phys. Med. Biol. 40, 295 (1995).

${ }^{20}$ P. Mannheimer, J. Casciana, M. Fein, and S. Nierlich, IEEE Trans. Biomed. Eng. 44, 148 (1997).

${ }^{21}$ E. Okada, M. Firbank, M. Schweiger, S. Arridge, M. Cope, and D. Deply, Appl. Opt. 36, 21 (1997).

${ }^{22}$ J. de Kock, K. Reynolds, L. Tarassenko, and J. Moyle, J. Med. Eng. Technol. 15, 111 (1991).

${ }^{23}$ J. Bland and D. Altman, Lancet 327, 307 (1986).

${ }^{24}$ D. Altman and J. Bland, Statistician 32, 307 (1983).

${ }^{25}$ M. Taylor and J. Whitman, Anaesthesia 43, 229 (1988). 